\title{
Stochastic collective dynamics of charged-particle beams in the stability regime
}

(revision day : September 27, 2000)

\author{
Nicola Cufaro Petroni*, Salvatore De Martino ${ }^{\S}$, Silvio De Siena ${ }^{\S}$ and Fabrizio Illuminati ${ }^{\S}$ \\ * Dipartimento Interateneo di Fisica dell'Università e del Politecnico di Bari; \\ INFN Sezione di Bari and INFM Unità di Bari; \\ Via G. Amendola 173, 70126 Bari, Italy; \\ Email: cufaro@ba.infn.it; Web: http://www.ba.infn.it/ cufaro/homepage.html \\ $\S$ Dipartimento di Fisica dell'Università di Salerno; \\ INFM Unità di Salerno and INFN Sezione di Napoli, Gruppo collegato di Salerno; \\ Via S. Allende, I-84081 Baronissi (SA), Italy; \\ Email: demartino@sa.infn.it,desiena@sa.infn.it,illuminati@sa.infn.it
}

\begin{abstract}
We introduce a description of the collective transverse dynamics of charged (proton) beams in the stability regime by suitable classical stochastic fluctuations. In this scheme, the collective beam dynamics is described by time-reversal invariant diffusion processes deduced by stochastic variational principles (Nelson processes). By general arguments, we show that the diffusion coefficient, expressed in units of length, is given by $\lambda_{c} \sqrt{N}$, where $N$ is the number of particles in the beam and $\lambda_{c}$ the Compton wavelength of a single constituent. This diffusion coefficient represents an effective unit of beam emittance. The hydrodynamic equations of the stochastic dynamics can be easily recast in the form of a Schrödinger equation, with the unit of emittance replacing the Planck action constant. This fact provides a natural connection to the so-called "quantum-like approaches" to beam dynamics. The transition probabilities associated to Nelson processes can be exploited to model evolutions suitable to control the transverse beam dynamics. In particular we show how to control, in the quadrupole approximation to the beam-field interaction, both the focusing and the transverse oscillations of the beam, either together or independently.
\end{abstract}

PACS numbers: 02.50.Ey, 29.27.Bd, 41.75.Lx.

\section{INTRODUCTION}

Most of the studies on the dynamics of charged beams in particle accelerators are concerned with classical phenomena of nonlinear resonances as isolated sources of unstable behaviors. Following this line of thought, a general understanding of classical dynamical processes in particle accelerators has been reached in recent years [1]. However, the transverse coherent oscillations of the beam density and profile require, to be explained, some mechanism of local correlation and loss of statistical independence. This implies the need to treat all the interactions as a whole, and to introduce an effective collective dynamics. Moreover, the overall interactions between charged particles and machine elements are really nonclassical in the sense that out of the many sources of noise that are present, almost all are mediated by fundamental quantum processes of emission and absorption of photons. Therefore the collective effective descriptions of these processes could contain, in principle, some quantum signature [2].

Starting from the above considerations, different approaches to the collective dynamics of charged beams have been developed. Some of them, relying on the Fokker-Planck equation and the statistical effects on the dynamics of colliding beams, have become an established reference in treating the sources of noise and dissipation in particle accelerators by standard classical probabilistic techniques [3]. Other, more recent, approaches are instead based on suitable coarse grainings of the constitutive kinetic equations, and yield Schrödinger-like equations, with a thermal unit of emittance replacing the Planck action constant ("quantum-like approaches" to beam dynamics) [4].

In this paper we first of all exploit (classical) mechanical criteria of stability in order to establish a connection with the statistical fluctuations affecting the beam dynamics. In particular we deduce a phenomenological expression of the characteristic unit of action (emittance) which quantifies the amount of these fluctuations and which is ultimately 
related to the microscopic scales and to the number of particles. Explicitly, the resulting expression for the diffusion coefficient $\mathcal{E}$ in units of length (i.e. the transverse unit of emittance) turns out to depend both on the fundamental Compton wavelength $\lambda_{c}$ and on the number $N$ of particles constituting the beam through the simple, but nontrivial, formula $\mathcal{E}=\lambda_{c} \sqrt{N}$. Thus motivated, we model the collective beam dynamics by introducing suitable stochastic processes with long-range coherent correlations.

This kind of analysis considers the regime of stability of our dynamical systems. In this framework we study the intermediate, but physically relevant, regime of beam dynamics in which a balance is realized, on the average, between the energy dissipation and the external RF energy pumping. Therefore our approach differs crucially from the previous ones, based only on the Fokker-Planck equation, since in this regime the overall classical beam dynamics can be considered at the same time stochastic and time-reversal invariant.

This scenario hints to very interesting perspectives for the following reasons. First of all, classical stochastic dynamical systems with time-reversal invariance have been introduced and extensively studied in the context of Nelson stochastic mechanics [5]. They are by now a well understood subject, both from the physical and mathematical point of view. For instance, among the possible physical applications of this modelling it is worth noticing the recovering of the Titius-Bode law for planet orbits in the solar system obtained in reference [6]. The study of these dynamical systems is based on an extension of the variational principles of classical mechanics to include the case of a diffusive kinematics replacing the deterministic one [7]. This is remarkable since variational principles are a very powerful tool in the description of physical systems. In the present case the stochastic variational principle yields two coupled hydrodynamic equations, respectively for the density and for the forward velocity field, which provide an effective description of the transverse oscillations of the beam density in the regime of stability.

On the other hand it is also interesting to remark that the two nonlinear coupled hydrodynamic equations of the stochastic mechanics are equivalent to one linear equation of the form of a Schrödinger equation, with the Planck action constant replaced by the diffusion coefficient of the random kinematics. This fact connects our approach to the quantum-like approaches to beam dynamics. Moreover, since this description involves not only a Fokker-Planck equation but also a dynamical prescription connected with an external potential, it allows to implement the powerful techniques of active control for the dynamics of the beam. This is at variance with the case of a purely dissipative Fokker-Planck dynamics which only describes a passive (irreversible) evolution of the state, and where you have no control whatsoever on the velocity field.

In fact, on the basis of the description of the beam collective dynamics in terms of the hydrodynamic equations of Nelson stochastic mechanics with the proper diffusion coefficient, we will show how we can implement techniques of control already developed in the general context of stochastic dynamical systems [8]. These techniques exploit the transition probabilities, a fundamental object in the theory of diffusion processes, in order to drive the beam toward a specified and controlled evolution. In particular in our quantum-like approach we construct time-dependent potentials which drive the system toward final states characterized by an improved collimation. At the same time, and independently, also the transverse betatron oscillations can be controlled and varied.

The paper is organized as follows: In Section II we exploit some basic criteria of mechanical stability in order to supply a phenomenological support for our fluctuative approach to collective dynamics of beams in particle accelerators. In particular these criteria will allow to connect the (transverse) emittance to the characteristic microscopic scale and to the total number of the particles in a bunch. In Section III we introduce a time-reversal invariant, stochastic description of the collective dynamics of the beam in the stability regime. The (hydrodynamic) equations of motion for the density and the profile of a bunch are here derived from a stochastic variational principle. In Section IV we sketch the general structure of controlled dynamics for quantum and quantum-like systems. In Section V we explicitly construct, as mentioned above, some examples of controlled beam evolution in the quadrupole approximation to the beam-field interaction. Finally in Section VI conclusions follow.

\section{COLLECTIVE BEHAVIOR OF DYNAMICAL SYSTEMS IN THE STABILITY REGIME}

Effective wave equations of the Schrödinger form, but associated to a non fundamental unit of action (that is, different from the Planck constant), have been introduced to describe the collective dynamics for classical physical systems with many degrees of freedom, including optical fibers [9] and charged particle beams in accelerators [4]. The basic feature common to all these systems is their high degree of coherence, which allows to introduce an effective description in terms of collective degrees of freedom. These represent the cooperative dynamical behavior of the many constituents of the system. This collective motion is summarized by the effective equations for the configurational (or phase-space) density and for the velocity fields.

Here we specialize to the dynamics of charged beams a general scheme previously introduced for the study of the dimensions of stability for macroscopic and mesoscopic systems [10]. The ultimate goal of the analysis of stability is 
to single out a (minimal) unit of action in terms of the characteristic constants, of the form of the interaction, and of the linear dimensions of the considered system. This analysis will allow us to relate the parameters associated to the collective degrees of freedom with the characteristics of the microscopic constituents. In Appendix A we summarize the procedure, while here we will just quickly quote the results.

We introduce a unit of action $\alpha$ (which will turn out to be minimal) defined by:

$$
\alpha=m \tilde{v}^{2} \tau,
$$

where $\tilde{v}$ denotes the characteristic mean velocity per particle in the system, while $\tau$ is a characteristic microscopic time whose size must be determined self-consistently. Imposing suitable criteria of stability, we obtain as first result [10] that the order of magnitude of this small time $\tau$ in (1) must be given by

$$
\tau \cong \frac{\mathcal{T}}{\sqrt{N}},
$$

where $\mathcal{T}$ is the macroscopic time scale associated to the entire system, and defined through the relation $\tilde{v}=R / \mathcal{T}$, where $R$ denotes the global length scale of the system. Therefore $\mathcal{T}$ has the meaning of a characteristic traveling time for a particle inside the system. Moreover, we obtain for the (minimal) unit of action $\alpha$ the general expression:

$$
\alpha \cong m^{1 / 2} R^{3 / 2} \sqrt{F(R)},
$$

where $F(R)$ denotes the value of the force ruling the system, computed on a distance scale of the order of magnitude of the global scale of the system [10].

It must be noted that the relation (2) has been originally conjectured by F. Calogero [11] in a different context. It is remarkable that, when tested in the range of all known stable macroscopic and mesoscopic aggregates of particles, the relation (3) always yields the order of magnitude of the Planck action constant [10]. This is the reason why we identify $\alpha$ as a minimal unit of action. It was not obviously trivial that from a purely mechanical criteria of stability the fundamental microscopic scale of action could emerge. Moreover this fact, together with the observation that we have self-consistently obtained the expression (2) for the microscopic time $\tau$, strongly hints to two further conclusions.

First, the factor $1 / \sqrt{N}$ in the scaling relation (2) typically hints to the presence of collective fluctuations, whose characteristic scale of time is given by $\tau$. This is not a surprising fact due to the large number of constituents in the system. Second, the universal coincidence of the minimal unit of action with the Planck action constant strongly points to the fact that these collective fluctuations are ultimately connected with the fundamental microscopic scales. It is worth noting that, as we will show later, this fact is not connected to complicated or mysterious effects of direct quantum origin, but it simply takes into account the constraint given by the characteristic spatial extension of the microscopic constituents.

In the specific instance of charged beams, we first verify the numerical coincidence of $\alpha$ with the Planck action constant. We then single out the expression of the (transverse) emittance in terms of the microscopic minimal length scale, and of the number of elementary constituents. This second step of our analysis is performed in the particular instance of proton beams since it would be impossible to find a classical characteristic length extension for the electron.

In the first step we consider a representative proton (electron), in the reference frame comoving with the bunch. Confinement and stability for the transverse motion of the bunch arise from the many interactions both among its constituents and between the same constituents and the external focusing electromagnetic fields. It is well known that the net effect can be, in the first quadrupole approximation, summarized by a harmonic force of modulus $F(r) \cong K r$, where $K$ is the effective phenomenological elastic constant associated to the transverse dynamics. Then equation (3) yields

$$
\alpha \cong m^{1 / 2} R^{2} K^{1 / 2} .
$$

We can now estimate $\alpha$ by introducing, besides the proton or the electron mass, the experimental values for the transverse linear dimension $R$ and for the effective elastic constant $K$. We have [1] $K \cong 10^{-12} \mathrm{Nm}^{-1}$ (transverse oscillations of protons at Hera) and $K \cong 10^{-11} \mathrm{Nm}^{-1}$ (transverse oscillations of electrons in linear colliders), while $R \cong 10^{-7} \mathrm{~m}$ in both experimental situations. As a consequence, in both cases equation (4) yields $\alpha \cong h$. We have therefore reached our first goal.

We now move in the second step to single out the parameter associated to the stability of the system at the mesoscopic scale in the case of charged beams. This parameter is given in terms of a characteristic unit of (transverse) emittance. The emittance is a scale of action that measures the spread of the bunch in phase space. It can also be defined as a unit of equivalent temperature or, in configuration space, as a unit of length. It is clear that this quantity must depend on the characteristic scales and on the total number of the elementary components in the system. In 
the framework of our scheme we are able to provide, at least in order of magnitude, a quantitative estimate of this dependence.

We proceed as follows: in the regime of stability and of thermal equilibrium, that we explicitly consider, the emittance can be expressed as a unit of equivalent thermal action. We denote by $T$ the equivalent unit of equivalent temperature of the system (namely the unit of energy divided by the Boltzmann constant $k_{B}$ ), and we define the characteristic thermal unit of action associated to the system as $k_{B} T \mathcal{T}$, the product of the unit of thermal energy and of the characteristic global time. In our scheme, when $\alpha \cong \hbar$, the time $\tau$ connected to the microscopic scales can also be identified with the usual scale of time associated to a microscopic system at the equilibrium temperature $T$; hence

$$
\tau \cong \frac{h}{k_{B} T} .
$$

Using relation (2) we finally obtain the equivalent thermal unit of action, the transverse emittance $\epsilon$, in terms of the minimal action $\hbar$ and of the total number of particle $N$ :

$$
\epsilon \equiv \frac{k_{B} T \mathcal{T}}{2 \pi} \cong \hbar \sqrt{N} .
$$

Up to now our results hold both for protons and electrons. However the previous relation allows a more direct check if written in terms of characteristic units of length. We know however that electrons do not possess a finite characteristic length extension, while for protons we know that such a linear extension coincides, in order of magnitude, with the Compton wave length. Thus, specializing to protons, we can show that, at least in order of magnitude, the numerical value of the transverse dimension of the bunch is:

$$
\frac{\epsilon}{m c} \cong \lambda_{c} \sqrt{N}
$$

where the Compton wavelength $\lambda_{c}=\hbar / m c$ ( $m$ is the proton mass, and $c$ is the velocity of light).

We can now interpret equation (7) in the following, simple way: the (transverse) mean dimension of the beam at equilibrium is connected to the characteristic length scale $\lambda_{c}$ of its microscopic constituents through the scaling factor $\sqrt{N}$. This last peculiar form, in turn, suggests a fluctuation mechanism which stabilizes the system. As previously anticipated, the microscopic scales influence the system only through the minimal length scale, i.e. the length extension of the elementary constituents, without direct connections to more involved quantum effects.

Inserting in equation (7) the numerical data of the proton wave length and of the number of proton in typical accelerators [1], we obtain the experimental order of magnitude of the transverse dimension of the bunch $10^{-7} \div$ $10^{-8} m[10]$. On the basis of our phenomenological scheme, we introduce in the next section a quantitative stochastic description of beam dynamics in the stability regime. In the following, consistently with the analysis carried out so far, we take as diffusion coefficient, expressed in unit of length, for the stochastic kinematics the quantity (transverse emittance)

$$
\mathcal{E} \equiv \frac{\epsilon}{2 m c} \cong \frac{\lambda_{c}}{2} \sqrt{N}
$$

where the factor 2, which does not affect the order of magnitude, is introduced for later computational convenience.

\section{STOCHASTIC COLLECTIVE DYNAMICS IN THE STABILITY REGIME}

In this section we model the spatial fluctuations (associated to the diffusion coefficient (8)) via the random kinematics performed by a representative particle that oscillates, in a reference frame comoving with the bunch, around the closed ideal orbit. This representative particle is identified with the collective degree of freedom by letting the associated probability density coincide with the real density of particles in the bunch. This last step is achieved by suitably rescaling the normalization of the total number of particles. Before proceeding, we establish the notations according to the standard conventions.

We denote $\mathbf{r} \equiv(x, y)$ a point in the transverse section orthogonal to the beam direction. We then measure the time in unit of length through the arc length $s$ along the design orbit (curvilinear coordinate). We now consider the (two-dimensional) diffusion process $\mathbf{q}(s)$ which describes the motion of the representative particle and whose probability density coincides with the particle density of the bunch in the transverse direction. The evolution in the "time" $s$ of the process $\mathbf{q}$ is described by the Itô stochastic differential equation

$$
d \mathbf{q}(s)=\mathbf{v}_{(+)}(\mathbf{q}(s), s) d s+\sqrt{\mathcal{E}} d \mathbf{w}(s),
$$


where $\mathbf{v}_{(+)}$is the (forward) drift, $d \mathbf{w}(s) \equiv \mathbf{w}(s+d s)-\mathbf{w}(s)$ is the $\delta$-correlated time increment of the standard Wiener noise, and, as already anticipated, the diffusion coefficient is the characteristic transverse emittance. Equation (9) defines the random kinematics performed by the collective degree of freedom.

In the stability regime the energy lost by photonic emissions is regained in the RF cavities, and on average the dynamics is time-reversal invariant. We are thus in a situation in which there are both a random kinematics and time reversal invariance. Therefore the dynamics must be independently added to the kinematics (at variance with the purely dissipative Fokker-Planck case) by introducing a suitable stochastic least action principle [7]. The latter is obtained as a generalization of the variational principle of classical mechanics, by replacing the classical deterministic kinematics, $d \mathbf{q}_{c}(s)=\mathbf{v}_{c}(s) d s$, with the random diffusive kinematics of equation (9). The equations of motion thus obtained take the form of two coupled hydrodynamic equations describing the evolution in time of the beam density and of the velocity field of the beam profile. In the following we give a brief sketch of the stochastic variational method, and we introduce the coupled hydrodynamic equations, referring for details to reference [7].

Given the stochastic differential equation (9), one can associate to the diffusion process $\mathbf{q}(s)$ a probability density $\rho(\mathbf{r}, s)$, where $\mathbf{r} \equiv(x, y)$ denotes the transverse coordinates (the radial coordinate and the vertical coordinate). Besides the forward drift $\mathbf{v}_{(+)}(\mathbf{r}, s)$, we can define a backward drift $\mathbf{v}_{(-)}(\mathbf{r}, s)=\mathbf{v}_{(+)}(\mathbf{r}, s)-2 \mathcal{E}(\nabla \rho)(\mathbf{r}, s) / \rho(\mathbf{r}, s)$, with $\nabla \equiv\left(\partial_{x}, \partial_{y}\right)$. It is useful to introduce two new variables, $\mathbf{v}(\mathbf{r}, s)$ and $\mathbf{u}(\mathbf{r}, s)$, respectively the current and the osmotic velocity fields, defined as:

$$
\mathbf{v}=\frac{\mathbf{v}_{(+)}+\mathbf{v}_{(-)}}{2} ; \quad \mathbf{u}=\frac{\mathbf{v}_{(+)}-\mathbf{v}_{(-)}}{2}=\mathcal{E} \frac{\nabla \rho}{\rho} .
$$

The velocities in equation (10) have a transparent physical meaning: the current velocity $\mathbf{v}$ represents the global velocity of the density profile, being the stochastic generalization of the velocity field of a classical perfect fluid. On the other hand the osmotic velocity $\mathbf{u}$ is clearly of intrinsic stochastic nature, for it is a measure of the non differentiability of the stochastic trajectories, and it is related to the spatial variations of the density.

In order to establish the stochastic generalization of the least action principle, one introduces a mean classical action in strict analogy to the classical deterministic action. The main difficulty in the stochastic case is due to the non differentiable character of the sample paths of a diffusion process which does not allow to define the time derivative $\dot{\mathbf{q}}$ of the process. Such a definition is possible only in an average sense trough a suitable limit on expectations. The stochastic action is then defined as [7]

$$
A\left(s_{0}, s_{1} ; \mathbf{q}(\cdot)\right)=\int_{s_{0}}^{s_{1}} \lim _{\Delta s \rightarrow 0^{+}} \mathbf{E}\left[\frac{m}{2}\left(\frac{\Delta \mathbf{q}}{\Delta s}\right)^{2}-V(\mathbf{q})\right] d s,
$$

where $\mathbf{E}(\cdot)=\int d r(\cdot) \rho(\mathbf{r}, s)$ denotes the expectation of functions of the process with respect to the probability density, $V$ denotes an external potential, and $\Delta \mathbf{q}(s)=\mathbf{q}(s+\Delta s)-\mathbf{q}(s)$. It can be shown that the mean action (11) associated to the diffusive kinematics (9) can be recast in the following particularly appealing Eulerian hydrodynamic form [5]:

$$
A\left(s_{0}, s_{1} ; \mathbf{v}, \rho\right)=\int_{s_{0}}^{s_{1}} d s \int d \mathbf{r}\left[\frac{m}{2}\left(\mathbf{v}^{2}-\mathbf{u}^{2}\right)-V(\mathbf{r})\right] \rho(\mathbf{r}, s),
$$

where $\mathbf{v}$ and $\mathbf{u}$ are defined in equation (10). The stochastic variational principle now follows by imposing the stationarity of the stochastic action $(\delta A=0)$ under smooth and independent variations $\delta \rho$ of the density, and $\delta \mathbf{v}$ of the current velocity, with vanishing boundary conditions at the initial and final times.

As a first consequence we get that the current velocity has a gradient form:

$$
m \mathbf{v}(\mathbf{r}, s)=\nabla S(\mathbf{r}, s),
$$

while the non linearly coupled Lagrange equations of motion for the density $\rho$, and for a current velocity $\mathbf{v}$ of the form (13) are: the continuity equation typically associated to every diffusion process

$$
\partial_{s} \rho=-\nabla \cdot(\rho \mathbf{v}),
$$

and a dynamical equation

$$
\partial_{s} S+\frac{m}{2} \mathbf{v}^{2}-2 m \mathcal{E}^{2} \frac{\nabla^{2} \sqrt{\rho}}{\sqrt{\rho}}+V(\mathbf{r}, s)=0,
$$

which characterizes the particular class of time-reversal invariant diffusion processes (Nelson processes). Last equation has the same form of the Hamilton-Jacobi-Madelung (HJM) equation, originally introduced in the hydrodynamic 
description of quantum mechanics by Madelung [12]. It can also be shown that the continuity equation (14) is equivalent to the standard Fokker-Planck equation

$$
\partial_{s} \rho=-\nabla \cdot\left[\mathbf{v}_{(+)} \rho\right]+\mathcal{E} \nabla^{2} \rho,
$$

by simple substitution from (10). The time-reversal invariance is assured by the fact that the forward drift velocity $\mathbf{v}_{(+)}(\mathbf{r}, s)$ is not a field given a priori, as usual for diffusion processes of the Langevin type; instead it is dynamically determined at any instant of time, starting by an initial conditions, through the HJM evolution equation (15).

The equations (14) and (15) describe the collective behaviour of the bunch at each instant of time through the evolution of both the particle density and the velocity field of the bunch. In particular we can calculate the expectations $\mathbf{E}[\mathbf{q}(s)]$ and $\mathbf{E}[\mathbf{v}(\mathbf{q}(s), s)]$, which supply the coordinates and the velocity components of the center of the bunch profile at time $s$, while the variances $\mathbf{V}\left[q_{i}(s)\right] \equiv \sqrt{\mathbf{E}\left[q_{i}^{2}(s)\right]-\mathbf{E}^{2}\left[q_{i}(s)\right]}$ represent the spreading of the bunch density along each space direction.

It is finally worth noticing that, introducing the trivial representation [12]

$$
\psi(\mathbf{r}, s)=\sqrt{\rho(\mathbf{r}, s)} \mathrm{e}^{i S(\mathbf{r}, s) / 2 m \mathcal{E}},
$$

the coupled equations (14) and (15) are equivalent to a single linear equation of the form of the Schrödinger equation in the function $\psi$, with the Planck action constant replaced by the emittance $\mathcal{E}$ :

$$
i 2 m \mathcal{E} \partial_{s} \psi=-2 m \mathcal{E}^{2} \nabla^{2} \psi+V \psi .
$$

In this formulation the "wave function" $\psi$ carries the information on both the dynamics of the bunch density $\rho$, and of the velocity field of the bunch, where the velocity field is determined through equation (13) by the phase function $S(\mathbf{r}, s)$. This shows, as previously claimed, that our procedure, starting from a different point of view, leads to a description formally analogous to that of the quantum-like approaches to beam dynamics [4].

\section{CONSTRUCTION OF CONTROLLED STATES FOR QUANTUM AND QUANTUM-LIKE SYSTEMS}

In the previous section we have introduced two coupled equations that describe the dynamical behaviour of the beam: the first is the Itô equation (9), or equivalently the Fokker-Planck equation (16); the second is the HJM equation (15). Here, we briefly sum up (with the present notations) a general procedure exploited in reference [8] to control the dynamics of quantum and quantum-like systems, while in the next section we will give an explicit application of the method to the transverse beam dynamics. From now on we will consider one-dimensional processes denoting by $\xi$ a one-dimensional space variable, in suitable units. In the next section the variable $\xi$ will be one of the transverse space coordinates. In reference [8], it has been shown that given a pair of functions $\rho(\xi, s)$ and $v_{(+)}(\xi, s)$ (density and forward velocity) which satisfy (16) or equivalently (14), the equation (15) with the given functions allows one to compute a control potential $V_{c}$. Remark that $\rho(\xi, s)$ and $v_{(+)}(\xi, s)$ can also be an entire class of functions of a given form.

Let us take for instance the solution $\rho(\xi, s)$ of a Fokker-Planck equation $(16)$ with a given $v_{(+)}(\xi, s)$ and a constant diffusion coefficient $\mathcal{E}$, define the function $W(\xi, s)$ from

$$
m v_{(+)}(\xi, s)=\partial_{\xi} W(\xi, s)
$$

and remind from (10) and (13) that the relation

$$
m v_{(+)}=\partial_{\xi}(S+\mathcal{E} \ln \tilde{\rho})
$$

must hold, where $\tilde{\rho}$ is the adimensional function (argument of a logarithm) obtained from the probability density $\rho$ by means of a suitable and arbitrary multiplicative constant with the dimensions of $\xi$. Hence from (19) and (20) we obtain for the phase function

$$
S(\xi, s)=W(\xi, s)-m \mathcal{E} \ln \tilde{\rho}(\xi, s)-\theta(s),
$$

which allows to determine $S$ from $\rho$ and $v_{(+)}$up to an additive arbitrary function of time $\theta(s)$. The functions $\rho$ and $S$, satisfying our kinematical relations (16), are a solution of our dynamical problem if they also satisfy the HJM equation (15). Since $S$ and $\rho$ are now fixed, this equation must be considered as a (constraint) relation defining a controlling potential $V_{c}$ which, after straightforward calculations, turns out to be of the form: 


$$
V_{c}(\xi, s)=m \mathcal{E}^{2} \partial_{\xi}^{2} \ln \tilde{\rho}+m \mathcal{E}\left(\partial_{s} \ln \tilde{\rho}+v_{(+)} \partial_{\xi} \ln \tilde{\rho}\right)-\frac{m v_{(+)}^{2}}{2}-\partial_{s} W+\dot{\theta}(s) .
$$

When the density $\rho$ interpolates between an initial and a final distribution, then the controlling potential $V_{c}$ interpolates between the corresponding initial and final potential. It is worth noticing that for a class of velocities $v_{(+)}$ (i.e. the non singular, time-independent velocities, but also particular instances of time-dependent velocities) the Fokker-Planck equation (16) alone would drive the density towards an asymptotic solution which does not depend on the initial condition (for details see [8] and [13]): this kind of evolution is not controlled by an external potential. In the time-independent case the asymptotic solution is also a stationary one. On the other hand, when the FokkerPlanck equation (16) is coupled with the dynamical HJM equation (15) we have a way to control the evolution and the right potential has the form (22) which depends on the velocity $v_{(+)}$. This method can in principle be applied to very complicated systems: for instance in the beam dynamics we could keep the beam coherent even in the presence of aberrations. However this problem is non explicitly solvable in closed form and requires some approximate treatment. At present we consider only the more simple, but still nontrivial, case of the quadrupole approximation to the beam-field interaction. In this case we can exactly compute controlling, quadratic potentials which drive the bunch to a final state with better focusing. Moreover we can avoid a technical difficulty present in the more general situation. Actually the general procedure often implies an initial singular behaviour in the phase function. In fact, when we suddenly impose to the initial state the forward drift associated to the final state, the new phase turns out to be "wrong" with respect to the initial density. Hence a "kick" in the potential is needed in order to produce such a sudden change in the phase. This fact shows that to be physically meaningful our procedure requires some smoothing. In [8], however, it was noted that, at least for a Gaussian choice of the initial and final densities, it is particularly simple to implement transitions which do not need any smoothing procedure. We can adopt this especially simple solution exactly in the case of beam dynamics in the quadrupole (harmonic potential) approximation.

\section{CONTROLLED BEAM DYNAMICS IN THE QUADRUPOLE APPROXIMATION}

We now move on to construct explicit examples of controlled beam dynamics. In considering an accelerating machine we assume, as usual, that the longitudinal and the transverse dynamics can be deemed independent with a high degree of approximation. We will work in the framework of the quadrupole approximation, with the further simplification of considering decoupled evolutions along the radial direction $x$ and the vertical direction $y$ in the local reference frame.

Under these conditions, we can split the original, two-dimensional diffusion process into two independent, onedimensional processes respectively along $x$ and $y$, each ruled by a harmonic potential. The configurational variable $\xi$ of the previous section can here indifferently be either $x$ or $y$ depending on the considered transverse direction. The potential in each transverse direction will have the general form:

$$
V(\xi, s)=\frac{1}{2} m \omega^{2}(s) \xi^{2}-m f(s) \xi+m U(s) .
$$

We have considered here a time-dependent frequency (parametric oscillator) in order to describe also the effects due to strong focusing [1]. Note that here we have a potential measured in units of mass, consistently with the choice of measure units made in the Section II. Our aim is now to exploit the hydrodynamic equations (14) and (15) as control equations for the beam dynamics. In particular, we will show how to compute a controlling, time-dependent potential which allows to drive a bunch prepared in a state with a certain degree of collimation towards a final state with better focusing.

We consider a Gaussian shape for the initial density profile of a bunch in each transverse direction, with constant dispersion, and with the centre of the profile which performs a classical harmonic motion with the same frequency associated to the initial potential (23). The motion of the centre models the betatron oscillations of the bunch. In our quantum-like approach, the state of the bunch is thus formally represented by a coherent state. As anticipated at the end of the previous section, we will now consider an instance of controlled evolution that does not require an extra smoothing procedure for the driving velocity field, i.e. the transition between pairs of Gaussian densities. In particular we will describe transitions from a coherent oscillating packet to another Gaussian state with a better collimation (smaller dispersion). It is worth noticing that we can also implement a procedure that allows to vary independently the dispersion (collimation) of the bunch density and the motion of the centre of the density profile (characteristics of the betatron oscillations).

To this end we will recall [14] that if the velocity field of a Fokker-Planck equation (16) with constant diffusion coefficient $\mathcal{E}$ (the transverse emittance) has the linear form $v_{(+)}(\xi, s)=A(s)+B(s) \xi$, with $A(s)$ and $B(s)$ continuous 
functions of $s$, then there are always Gaussian solutions $\mathcal{N}(\mu(s), \nu(s))$, where $\mu(s)$ (the displacement of the centre of the Gaussian distribution) and $\nu(s)$ (the variance of the Gaussian distribution) are solutions of the differential equations

$$
\mu^{\prime}(s)-B(s) \mu(s)=A(s) ; \quad \nu^{\prime}(s)-2 B(s) \nu(s)=2 \mathcal{E},
$$

with suitable initial conditions, and where the prime denotes the derivative with respect to $s$. As previously stated, all along the time evolution our states keep a Gaussian shape for the density, and the centre of the density profile performs an arbitrarily assigned motion. Then, if we adopt the concise quantum-like representation of the bunch state (17) it is straightforward to show that the general form for the wave packet will be:

$$
\psi(\xi, s)=\left(\frac{1}{2 \pi \nu}\right)^{1 / 4} \exp \left[-\frac{(\xi-\mu)^{2}}{4 \nu}+\frac{i}{2 m \mathcal{E}}\left(m \mu^{\prime} \xi+m \frac{\nu^{\prime}}{4 \nu}(\xi-\mu)^{2}+\theta\right)\right],
$$

while the forward velocity field reads

$$
v_{(+)}(\xi, s)=\mu^{\prime}+\frac{\nu^{\prime}-2 \mathcal{E}}{2 \nu}(\xi-\mu) .
$$

Here the $s$-dependent functions $\mu(s)$ and $\nu(s)$ describe respectively the motion of the centre of the density profile and the spreading of the bunch density in the chosen transverse direction; on the other hand $\theta(s)$ plays the role of an arbitrary integration constant as can be seen from (21). Of course a suitable potential must also be tailored from the equation (22) in order to keep the evolution of the wave function (25) on the right track: we will show that in fact this control potential has the form suggested in (23).

The equation (25) represents the most general Gaussian packet, with a given generic motion $\mu(s)$ of its centre and with a given dispersion $\nu(s)$, associated to a linear form of the forward velocity in the Fokker-Planck equation (16). This also allows us to keep independent the initial and the final motion of the centre of the packet from the dispersion. As a first example let us now consider the transitions between two states of the form (25) with constant dispersion and with a harmonic motion of the centre of the profile. If initially (namely for $s \ll \tau$, where from now on $\tau$ is the transition instant) we start with $\nu(s)=\nu_{1}$ and $\mu(s)=a_{1} \cos \left(\omega_{1} s\right)$, we will have an initial Gaussian density profile with spreading $\nu_{1}$ and with harmonic betatron oscillation of frequency $\omega_{1}=\mathcal{E} / \nu_{1}$. We now want to drive the system towards a final (for $s \gg \tau$ ) state of the form (25), but with a spreading $\nu_{2}<\nu_{1}$ (better collimation) and a new betatron oscillation $\mu_{2}(s)$. To this end we only need to put in the solution $\mathcal{N}(\mu(s), \nu(s))$ two functions $\mu(s), \nu(s)$ which interpolate between the corresponding initial and final functions of the motion of the centre, and of the spreading respectively. Moreover, with a suitable choice of the $\xi$-independent part of the phase function in (25), the forward velocity field will also smoothly interpolate between the initial and the final velocity fields [8]. The control potential which drives the solution toward the required end is finally obtained by the equation (22) with $\tilde{\rho}$ given by the interpolating solution $\mathcal{N}(\mu(s), \nu(s))$, and with $v_{(+)}$given by the associated forward velocity. Of course there is a large number of possible choices for the interpolating functions $\mu(s), \nu(s)$ : this will allow us to single out the forms that better realize specific requirements. For example, it is possible to choose a characteristic transition time (the time needed to go from the initial to the final state) by inserting exponential relaxation terms in the interpolating functions.

We will now supply a few explicit examples of transitions. Our initial $(s \ll \tau)$ Gaussian, coherent, oscillating wave function has the form

$$
\psi_{1}(\xi, s)=\left(\frac{1}{2 \pi \nu_{1}}\right)^{1 / 4} \exp \left[\frac{-\left(\xi-a_{1} \cos \omega_{1} s\right)^{2}}{4 \nu_{1}}-i \frac{4 a_{1} \xi \sin \omega_{1} s-a_{1}^{2} \sin 2 \omega_{1} s+4 \nu_{1} \omega_{1} s}{8 \nu_{1}}\right],
$$

where we must also remember that

$$
\omega_{1}=\frac{\mathcal{E}}{\nu_{1}}
$$

The relation (28) means that our initial potential is purely harmonic with frequency $\omega_{1}$. By comparing (27) with the required Gaussian density, i.e. imposing that

$$
\left|\psi_{1}(\xi, s)\right|^{2}=\rho(\xi, s)=\frac{\mathrm{e}^{-(\xi-\mu(s))^{2} / 2 \nu(s)}}{\sqrt{2 \pi \nu(s)}}
$$

we get the initial identification 


$$
\mu(s)=a_{1} \cos \omega_{1} s=a_{1} \cos \left(\frac{\mathcal{E} s}{\nu_{1}}\right), \quad \nu(s)=\nu_{1}, \quad(s \ll \tau) .
$$

As for the initial phase function, by inspection of equations (27) and (17), and by taking (28) into account, we immediately get

$$
S(\xi, s)=m \omega_{1}\left(\frac{a_{1}^{2}}{4} \sin 2 \omega_{1} s-\mathcal{E} s-a_{1} \xi \sin \omega_{1} s\right), \quad(s \ll \tau) .
$$

First of all we want to describe the (smooth) transition of our initial wave function to a final one of the same form but characterized by a new set of parameters:

$$
a_{1} \rightarrow a_{2}, \quad \nu_{1} \rightarrow \nu_{2}, \quad \omega_{1}=\frac{\mathcal{E}}{\nu_{1}} \rightarrow \omega_{2}=\frac{\mathcal{E}}{\nu_{2}}
$$

The choice (32) means that also the final potential is still purely harmonic, but with a new frequency $\omega_{2}$. In order to achieve that we consider for example the function

$$
\Gamma(s)=\frac{1}{1+\mathrm{e}^{-(s-\tau) / \gamma}}
$$

which smoothly goes from 0 (for $s \ll \tau$ ) to 1 (for $s \gg \tau$ ) with a flex point in $s=\tau$ and a transition velocity equal to $1 / \gamma$. Of course here $\tau$ and $\gamma$ are completely free parameters: a suitable choice of them will allow to fine tune the timing and the velocity of the transition. Now the required transition is implemented by choosing

$$
\mu(s)=a_{1} \cos \left(\frac{\mathcal{E} s}{\nu_{1}}\right)(1-\Gamma(s))+a_{2} \cos \left(\frac{\mathcal{E} s}{\nu_{2}}\right) \Gamma(s), \quad \nu(s)=\nu_{1}(1-\Gamma(s))+\nu_{2} \Gamma(s),
$$

which realizes (32) and hence interpolates between the two initial and final Gaussian, coherent, oscillating states.

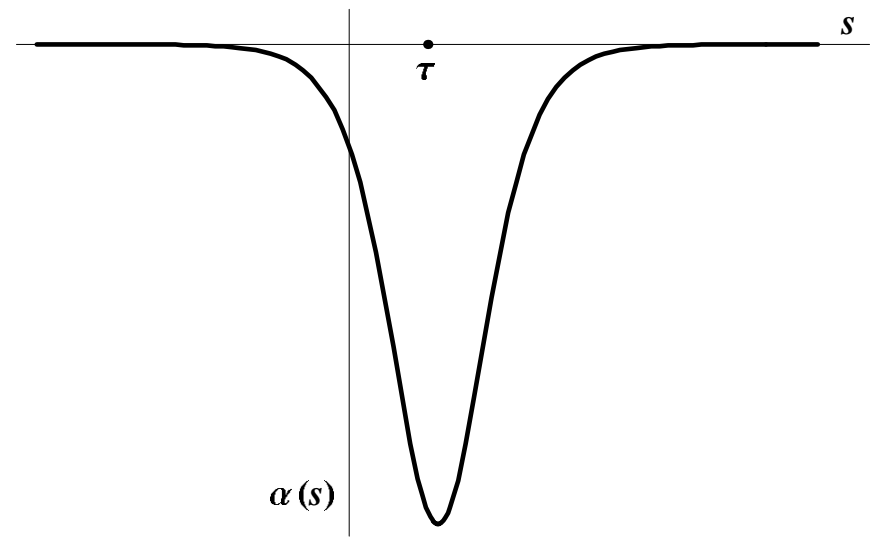

FIG. 1. The function $\alpha(s)$ is the coefficient of the $\xi^{2}$ term in the phase function (35). Notice that it goes quickly to zero, as required, outside the transition region of width $\gamma$ around $s=\tau$. Its negative values are due to the choice of monotonically decreasing dispersion $\nu$ (better collimation).

The phase function can now be calculated from (25) and we have

$$
\begin{aligned}
S(\xi, s) & =m\left[\alpha(s) \xi^{2}+\beta(s) \xi+H(s)+\theta(s)\right] \\
\alpha(s) & =\frac{\nu^{\prime}}{4 \nu}, \quad \beta(s)=\mu^{\prime}-\frac{\mu \nu^{\prime}}{2 \nu}, \quad H(s)=\frac{\nu^{\prime} \mu^{2}}{4 \nu} .
\end{aligned}
$$

Since $\alpha, \beta$ and $H$ are now fixed by (34), a comparison between (35) and (31), and in particular between the asymptotic $(s \rightarrow \pm \infty)$ expressions of the $\xi$-independent term of the phase, will suggest the following form for the arbitrary $\theta(s)$ function:

$$
\theta(s)=\left[\frac{\mathcal{E} a_{1}^{2}}{4 \nu_{1}} \sin \left(\frac{2 \mathcal{E} s}{\nu_{1}}\right)-\frac{\mathcal{E}^{2} s}{\nu_{1}}\right](1-\Gamma(s))+\left[\frac{\mathcal{E} a_{2}^{2}}{4 \nu_{2}} \sin \left(\frac{2 \mathcal{E} s}{\nu_{2}}\right)-\frac{\mathcal{E}^{2} s}{\nu_{2}}\right] \Gamma(s)-H(s) .
$$


Finally the potential will have the form

$$
\begin{aligned}
V_{c}(\xi, s) & =m\left[\frac{1}{2} G(s) \xi^{2}-F(s) \xi+W(s)\right] \\
G(s)=\frac{\mathcal{E}^{2}}{\nu^{2}}-\frac{\nu^{\prime \prime}}{2 \nu}+\frac{\nu^{\prime 2}}{4 \nu^{2}}, \quad F(s) & =\mu^{\prime \prime}+\mu G, \quad W(s)=\frac{G \mu^{2}}{2}-\frac{\mu^{\prime 2}}{2}-\frac{\mathcal{E}^{2}}{\nu}-\theta^{\prime}(s),
\end{aligned}
$$

where now all the terms are given by the previous relations.

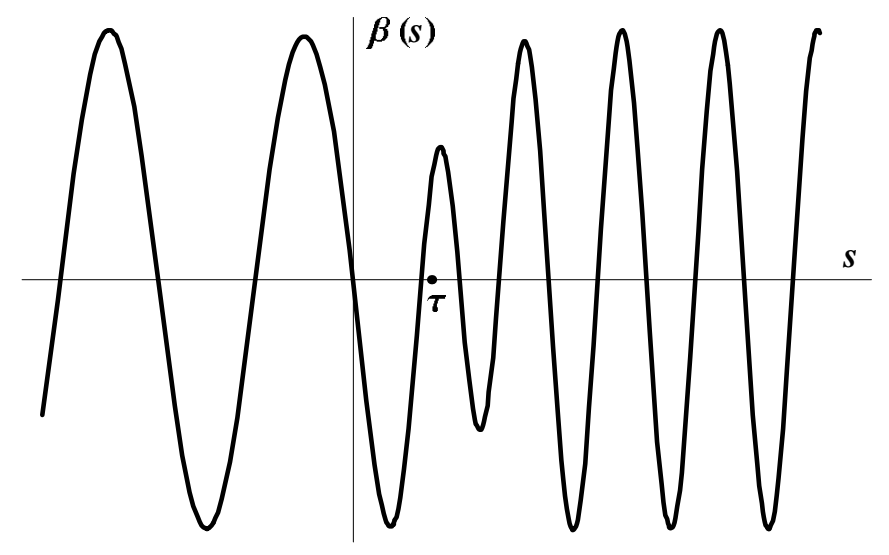

FIG. 2. The function $\beta(s)$ is the coefficient of the $\xi$ term in the phase function (35). Outside the transition region it oscillates, as required, with stable frequencies: $\omega_{1}$ for $s \ll \tau$, and $\omega_{2}$ for $s \gg \tau$. The faster oscillation for $s \gg \tau$ is due to the fact that $\omega_{2}>\omega_{1}$.

As already remarked this potential has exactly the form (23). The functions $\alpha(s), \beta(s), G(s), F(s)$ and $W(s)$, which determine the potential, can now be explicitly calculated for our example from the equations (34). Their analytic expressions are by far too long (albeit elementary), however their graphical behaviour is very simple and can be easily plotted. In particular see the Figures 1 - 5 for a few typical diagrams displaying the principal characteristics of these parameters which completely define the transition.

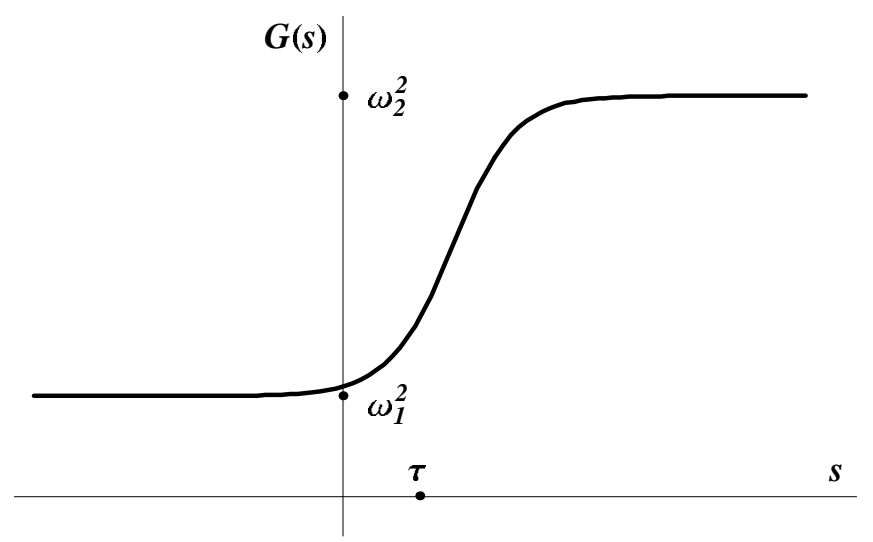

FIG. 3. The function $G(s)$ represents the square of the time-dependent frequency of the harmonic, controlling potential (38). Reminding equation (32) and that $\nu_{2}<\nu_{1}$ we have $\omega_{2}>\omega_{1}$.

First of all the functions $\alpha$ and $\beta$ show the behaviour of the phase function: remark that it is not necessary to produce a plot for the $\xi$-independent part of the phase since the relation (37) by definition imposes the right asymptotic behaviour. Figure 1 shows that $\alpha(s)$ has a smooth extremal value around the transition at $\tau$, while it also quickly goes to zero for $s \ll \tau$ and $s \gg \tau$ : hence no terms depending on $\xi^{2}$ remain asymptotically in the phase as required by the form (27). On the other hand Figure 2 shows that $\beta(s)$ asymptotically has a sinusoidal behaviour with different amplitudes and frequencies in the two zones $s \ll \tau$ and $s \gg \tau$ : this also is in good agreement with the required form of the phase. 


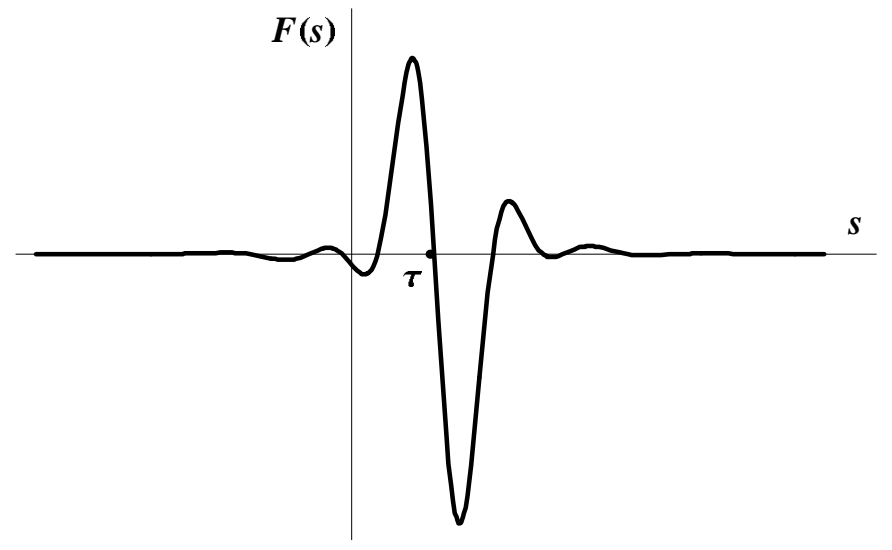

FIG. 4. The function $F(s)$ represents the time-dependent coefficient of the $\xi$ term in the harmonic, control potential (38). The fact that it quickly goes to zero outside the transition region is a consequence of the relation $\omega_{2}=\mathcal{E} / \nu_{2}$ and of the choice (37) for the function $\theta(s)$.

As for the control potential, Figure 3 indicates that $G(s)$, which represents the parameter of the harmonic part (depending on $\xi^{2}$ ) of $V_{c}$, smoothly goes from $\omega_{1}^{2}$ to $\omega_{2}^{2}$ along the transition and sticks to these two constant values outside the transition zone. From Figure 4 and Figure 5 we finally see that $F(s)$ and $W(s)$, which are respectively the coefficient of the linear part and of the $\xi$-independent term in the control potential, are different from zero only around the transition at $s=\tau$, while they are everywhere zero far away from $\tau$. As a consequence also the potential $V_{c}$ has the required time behaviour since it is a simple harmonic potential for $s \ll \tau$ and $s \gg \tau$ (albeit with two different frequencies), and shows some extra terms only in a limited interval around the transition.

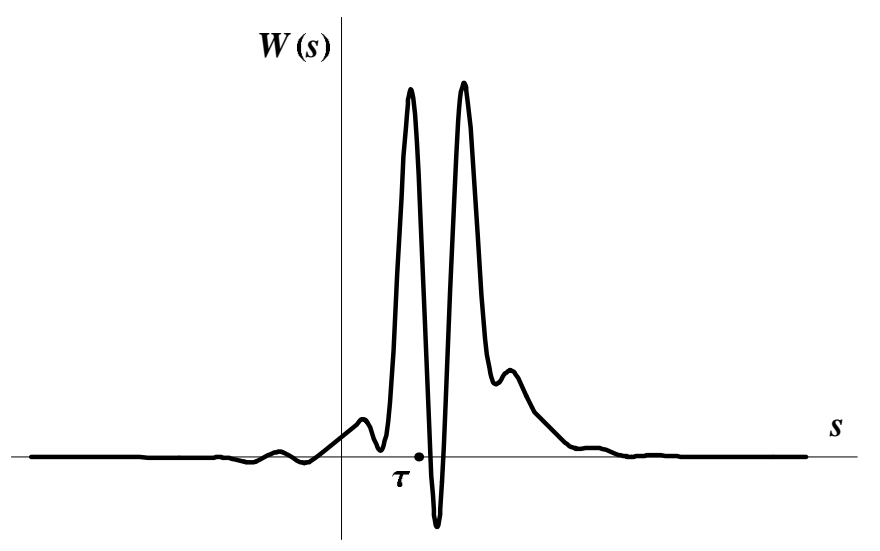

FIG. 5. The function $W(s)$ represents the coefficient of the $\xi$-independent term in the harmonic, control potential (38). Here too the fact that it quickly goes to zero outside the transition region is a consequence of the relation $\omega_{2}=\mathcal{E} / \nu_{2}$ and of the choice (37) for the function $\theta(s)$.

Of course this does not constitute the only potential we can obtain by this way. For example the function $\mu(s)$, instead, could be chosen in such a way that the oscillation of the centre of the profile be slower than the initial one, despite the fact that the better collimation requires a final potential associated to a frequency $\omega_{2}=\mathcal{E} / \nu_{2}$ larger than the initial one and then to a faster betatron oscillation with the same amplitude. This can be achieved by keeping a suitable forcing part $F(s)$ different from zero also for $s \gg \tau$ : namely in this case the final potential does not reduces itself to a simple harmonic one. It is easy to show that if the final oscillation has the generalized form

$$
\mu(s)=a \cos (\omega s)+\frac{b}{m} \sin (\omega s)
$$

with $\omega$ not coincident with $\mathcal{E} / \nu$, the final forcing function $F(s)$ calculated from (38) will correspondingly be

$$
F(s)=m\left(\omega^{2}-\frac{\mathcal{E}^{2}}{\nu^{2}}\right)\left(a \cos \omega s+\frac{b}{m} \sin \omega s\right) .
$$




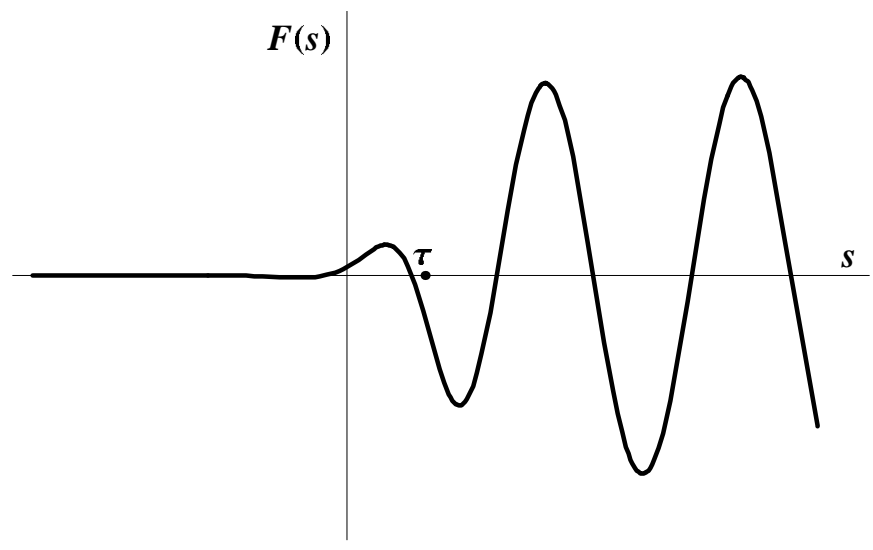

FIG. 6. This behaviour of the function $F(s)$, different from that of Figure 4 , is due to the fact that the relation $\omega_{2}=\mathcal{E} / \nu_{2}$ is no longer satisfied and the choice (43) is taken for the function $\theta(s)$. The non vanishing oscillations of $F(s)$ in the asymptotic region $s \gg \tau$ allow to reduce the otherwise naturally enhanced betatron oscillations.

In this case the potentials are more complicated but can still be suitably explored by means of our method. As an example we consider the case where the final state is characterized by two independent parameters: $\omega_{2}$ for the frequency and $\nu_{2}$ for the packet spreading. Now a relation similar to (28) will be no longer satisfied. As a consequence the choice (34) will be changed in

$$
\mu(s)=a_{1} \cos \left(\frac{\mathcal{E} s}{\nu_{1}}\right)(1-\Gamma(s))+a_{2} \cos \left(\omega_{2} s\right) \Gamma(s), \quad \nu(s)=\nu_{1}(1-\Gamma(s))+\nu_{2} \Gamma(s),
$$

while we get a new determination for the arbitrary $\theta(s)$ function:

$$
\theta(s)=\left[\frac{\mathcal{E} a_{1}^{2}}{4 \nu_{1}} \sin \left(\frac{2 \mathcal{E} s}{\nu_{1}}\right)-\frac{\mathcal{E}^{2} s}{\nu_{1}}\right](1-\Gamma(s))+\left[\frac{\omega_{2} a_{2}^{2}}{4} \sin \left(2 \omega_{2} s\right)-\mathcal{E} \omega_{2} s\right] \Gamma(s)-H(s) .
$$

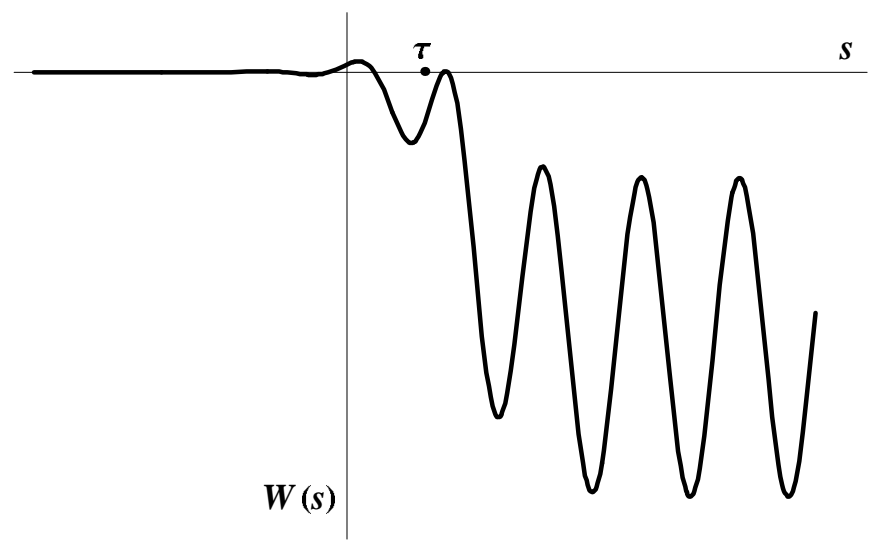

FIG. 7. Here too, as for the function $F(s)$, the new behaviour of the function $W(s)$, different from that of Figure 5 , is due to the fact that the relation $\omega_{2}=\mathcal{E} / \nu_{2}$ is no more satisfied and the choice (43) is taken for the function $\theta(s)$.

The functions defining the time evolution of both the phase and the potential can now be calculated once more and we find that, the functions $\alpha(s), \beta(s)$ keep a form very similar to the previous one. Instead the new $G(s)$ displays an opposite behaviour with respect to the Figure 3: in this case the final frequency $\omega_{2}$ is smaller than the initial frequency $\omega_{1}$, and thus the betatron oscillations are suppressed. On the other hand the shape is still of the form of a sigmoid. As for the functions $F(s)$ and $W(s)$ they show a different asymptotic behaviour as can be seen from Figure 6 and 7 . 


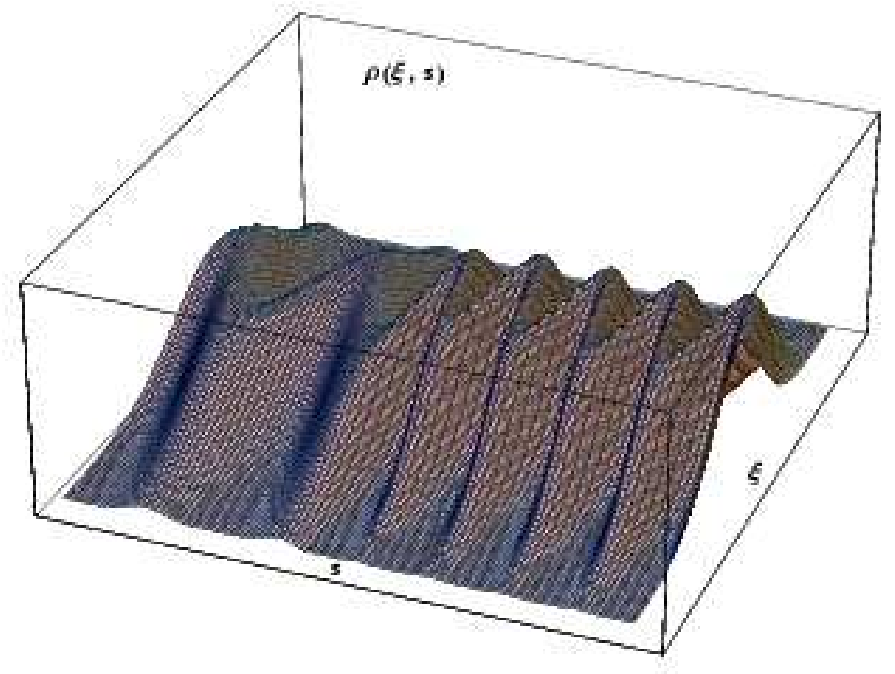

FIG. 8. The density (29) of the bunch as a function of $s$ and $\xi$. Here the squeezing is performed with the constrains (32) between the parameters $\omega$ and $\nu$, and hence without control on the betatron oscillations. Hence these oscillations are enhanced as a consequence of the squeezing.

In particular we see that, as predicted, $F(s)$ and $W(s)$ no more disappear for $s \gg \tau$, so that asymptotically we do not have a purely harmonic potential since now in (23) both the term linear and that constant in $\xi$ will be present for every $s>\tau$. However it is clear that other choices are always possible: for example the arbitrary function $\theta(s)$ could be defined so that in (38) the $\xi$-independent term $W(s)$ of the potential $V_{c}$ be identically zero. Of course there would be a price to pay for that: in fact now in the phase function $S$ the $\xi$-independent term will no more follow an asymptotic behaviour of the type (31) since the relation (43) will no more be satisfied.

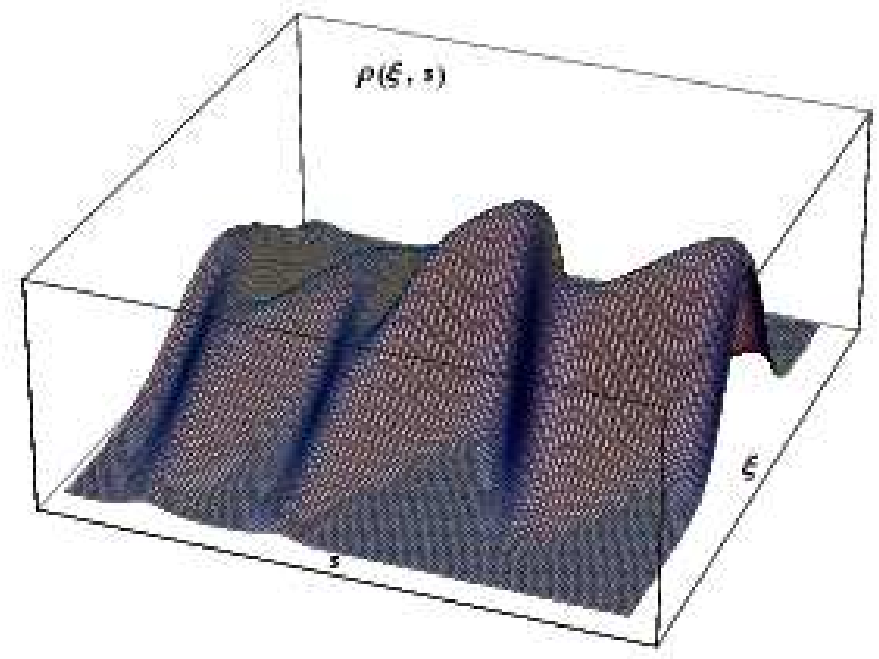

FIG. 9. At variance with Figure 8, here the density (29) is squeezed with no constrains of the type (32) between the parameters $\omega$ and $\nu$. As a consequence we were able to slow down at the same time also the betatron oscillations.

In the most general case of transitions between states with non constant dispersion (strong focusing) it is clear that the procedure can also be suitably extended. In fact it is sufficient to exploit for instance the expression (34) for the interpolating dispersion, but with time dependent initial and final dispersions $\nu_{1}(s)$ and $\nu_{2}(s)$. The general form (38) of the controlling potential is thus calculated, but with a new expression for $\nu(t)$. Finally, also the initial and final laws of motion of the profile centre, $\mu_{1}(s)$ and $\mu_{2}(s)$, can always be chosen as in the previously discussed example. However, in this case, a forcing part $F(s)$ is needed to retain the oscillatory motion (40) for $s \gg \tau$. In conclusions Figures 8 and 9 show the $s$-evolution of the density of the bunch. Both describe a squeezing of the beam, but Figure 8 reproduces the case where the frequency of the betatron oscillation is enhanced, while Figure 9 is related to the case where these oscillations are reduced. 


\section{CONCLUSIONS}

In the first part of this paper we have applied to the collective dynamics of beams in particle accelerators a stability analysis already developed for general particle systems. This analysis has allowed us to single out scaling factors relating the parameters ruling the collective dynamics in the beams with the microscopic scales.

In the second part of the paper we have considered the stability regime of a beam, in which the energy loss due to the radiation damping is on average compensated by the external RF energy pumping. The collective beam dynamics in this regime is described by time-reversal invariant diffusion processes (Nelson processes) which are obtained by a stochastic extension of the least action principle of classical mechanics. The choice of the diffusion coefficient is dictated by the unit of emittance determined in the first part of the paper. The collective dynamics of beams is then described by two non-linearly coupled hydrodynamic equations. It has also been observed that the linearization of these equations connects this approach to a Schrödinger-like (quantum-like) effective description of the beam dynamics previously developed through different approaches.

In the last part of the paper we have shown that the transition probabilities of Nelson processes can be exploited to control the collimation and the oscillations of the beam in the quadrupole approximation, both in the weak focusing and in the strong focusing regimes. In this framework we have explicitly computed the controlling potentials that realize some relevant controlled evolutions. The controlling potentials can be engineered by suitable tuning of the external RF and magnetic fields. We have considered evolutions that drive the beam from a less collimated to a better collimated state. We have furthermore shown that this goal can also be achieved without increasing the frequency of the betatron oscillations which can in fact be independently controlled during the evolution. In the forthcoming papers we will study the extension of these control techniques beyond the quadrupole approximation and address in detail applications to existing machines, problems related to dynamical instabilities and topics about the halo formation, a problem which has recently been addressed in the framework of a quantum-like approach [16].

\section{APPENDIX A: MINIMAL ACTION AND SCALING FACTORS FOR STABLE SYSTEMS}

We consider a generic stable system confined in a region of space of linear dimension $R$, constituted by a large number $N$ of identical particles of mass $m$, and ruled by an attractive classical (possibly effective) law of force $F(r)$. We introduce a unit of action $\alpha$ (which will turn out to be minimal) by the following relation:

$$
\alpha=m \tilde{v}^{2} \tau \text {. }
$$

In this equation, $\tilde{v}$ denotes the characteristic mean velocity per particle in the system, while $\tau$ is a characteristic microscopic time whose size must be self-consistently determined (for details see reference [10]). In order to obtain an explicit expression for $\alpha$, we then impose the following criteria of stability: firstly, we require that the characteristic potential energy of each particle be on average equal to its characteristic kinetic energy (virial theorem):

$$
\mathcal{L} \cong m \tilde{v}^{2},
$$

where $\mathcal{L}$ is the work performed in mean by the entire system on a single constituent. Then, if the system extends on the characteristic length scale $R$ we have, in order of magnitude

$$
\mathcal{L} \cong N F(R) R,
$$

where $F(R)$ is the force evaluated on a distance of the order of magnitude of the linear global dimension of the system. Relations (A2) and (A3) are now summarized by the following expression of the characteristic velocity $\tilde{v}$ :

$$
\tilde{v} \cong \sqrt{\frac{N F(R) R}{m}}
$$

We then define the macroscopic time scale $\mathcal{T}$ associated to the entire system, through the obvious relation $\tilde{v}=R / \mathcal{T}$ (therefore $\mathcal{T}$ has the meaning of a characteristic traveling time for a particle inside the system). We insert into equation (A1) both the latter expression and the expression (A4), obtaining the following form for the (minimal) unit of action:

$$
\alpha \cong \sqrt{m F(R)} R^{3 / 2} N^{1 / 2} \frac{\tau}{\mathcal{T}}
$$


We now introduce a second requirement for the mechanical stability, namely that due to the large number of particles, the unit of action $\alpha$ be not sensibly dependent on $N$. As a natural consequence, we are led to impose a relation between the microscopic characteristic time $\tau$ and the macroscopic characteristic traveling time $\mathcal{T}$ of the form

$$
\tau \cong \frac{\mathcal{T}}{\sqrt{N}}
$$

Finally, by inserting (A6) into equation (A5) we finally obtain the (minimal) unit of action

$$
\alpha \cong m^{1 / 2} R^{3 / 2} \sqrt{F(R)}
$$

[1] M. Conte and W. W. MacKay, An Introduction to the Physics of Particle Accelerators (World Scientific, Singapore, 1991); D. A. Edwards and M. J. Syphers, An Introduction to the Physics of High Energy Accelerators (Wiley, N. Y., 1993); H. Wiedemann, Particle accelerator physics (Springer, Berlin, 1998).

[2] N. Cufaro Petroni, S. De Martino, S. De Siena and F. Illuminati, in Quantum aspects of beam physics, P. Chen ed. (World Scientific, Singapore, 1999) p. 710;

N. Cufaro Petroni, S. De Martino, S. De Siena, R. Fedele, F. Illuminati and S. I. Tzenov, in Proceedings of the European particle accelerator conference - EPAC98, S. Myers et al. eds. (IoP Publishing, Bristol, 1998), p. 1259.

[3] S. Chattopadhyay, AIP Conf. Proc. 127, 444 (1983);

F. Ruggiero, Ann. Phys. (N.Y.) 153, 122 (1984);

J. F. Schonfeld, Ann. Phys. (N.Y.) 160, 149 (1985);

F. Ruggiero, E. Picasso and L. A. Radicati, Ann. Phys. (N. Y.) 197, 396 (1990).

[4] R. Fedele, G. Miele and L. Palumbo, Phys. Lett. A 194, 113 (1994), and references therein; S.I.Tzenov, Phys.Lett. A 232, 260 (1997).

[5] E. Nelson, Quantum Fluctuations (Princeton University Press, Princeton N. J., 1985); F. Guerra, Phys.Rep. 77, 263 (1981).

[6] S. Albeverio, Ph. Blanchard and R. Høgh-Krohn, Expo. Math. 4, 365 (1983).

[7] F. Guerra and L. M. Morato, Phys. Rev. D 27, 1774 (1983).

[8] N. Cufaro Petroni, S. De Martino, S. De Siena, and F. Illuminati, J. Phys. A 32, 7489 (1999).

[9] D. Marcuse, Light Transmission Optics (Van Nostrand, New York, 1972); J. A. Arnoud, Beam and Fiber Optics (Academic Press, New York, 1976).

[10] S. De Martino, S. De Siena, and F. Illuminati, Physica A 271, 324 (1999).

[11] F. Calogero, Phys. Lett. A 228, 335 (1997).

[12] E. Madelung, Z. Phys. 40, 332 (1926);

D. Bohm, Phys. Rev. 85, 166, 180 (1952).

[13] N. Cufaro Petroni, S. De Martino and S. De Siena, Phys. Lett. A 245, 1 (1998).

[14] N. Cufaro Petroni and F. Guerra, Found. Phys. 25, 297 (1995).

[15] K. Huang, Statistical mechanics, 2nd Ed. (Wiley, N. Y., 1987).

[16] S.A.Khan and M.Pusterla, Eur. Phys. J. A 7, 583 (2000). 